\title{
THE EJECTION OF QSOs FROM GALAXIES
}

\author{
G. Burbidge and A. Hewitt \\ Center for Astrophysics and Space Sciences \\ University of California, San Diego \\ La Jolla, California 92093
}

\section{INTRODUCTION}

In the 1950s, V.A. Ambartsumian (1958) proposed that galaxies result from explosive processes in galactic centers. Soon after the discovery of powerful radio galaxies in this same period, it became clear that explosive ejection of gas and relativistic particles was a common feature of active galaxies (Burbidge, Burbidge and Sandage 1963).

Later, conventional ideas were developed which attribute double radio sources to jets of relativistic particles which carry energy into the radio lobes where the particles are then reaccelerated by shock phenomena (Blandford and Rees 1974). These ideas have been developed in great detail. However, the alternative approach, that energy is released from explosive centers through the ejection of coherent objects (Burbidge 1967) has never been followed up, and the physics of such processes is not understood.

In this paper we shall outline the evidence that suggests that QSOs are ejected from bright, comparatively nearby galaxies, and also the evidence that suggests that radio galaxies are even more powerful sources of coherent objects.

\section{QSOs AND BRIGHT GALAXIES}

The evidence for much higher densities of QSOs near galaxies than in the field starts with the work of Arp and of Burbidge et al (1971)(see also Kippenhahn and de Vries (1974)). Over the last 20 years, Arp has found many more cases of this kind which are summarized in his book (Arp 1987). More recently the samples found by Stocke et al (1987) and the studies by Pocock et al $(1984)$ and Monk et al $(1986,1988)$ leave us in no doubt that there is an excess of QSOs near to bright galaxies, over that present in the general field. Some more references which strongly support this result are Burbidge $(1979,1981)$, Arp (1971), Sulentic (1983). Unless this is due to gravitational microlensing which we doubt, it is evidence for ejection. 


\section{THE ALIGNMENT OF RADIO SOURCES, OPTICAL EMISSION, GALAXIES AND QSOs}

When they were first discovered, one of the most striking features of the powerful extragalactic radio sources was their double nature, with an optical object in the center and two approximately symmetrical radio lobes lying along a major axis. As the radio sources have been further studied at progressively higher resolution and at many frequencies, this basic picture has been further amplified and confirmed. It has been shown that some sources are extremely large and can extend over more than $1 \mathrm{Mpc}$. It has also been shown that jets of non-thermal emission are sometimes present on much smaller scales, and that there is often rather precise alignment of such features over scales ranging from $\sim 100 \mathrm{pc}$ at the center up to $10^{\beta} \mathrm{pc}$. Such alignments clearly suggest ejection along an axis which has a memory. We have no direct evidence concerning what is ejected to give rise to the radio lobes, though we do know that the emission is incoherent synchrotron radiation. The most favored explanation is that put forward by Blandford and Rees (1974) who proposed the twin jet model which consists of a pair of jets of relativistic particles which feed energy into the radio lobes where it is used to reaccelerate the electrons to give the radio emission. An alternative to this model, which has not been followed up since it was first proposed is the model in which discrete self-gravitating objects are ejected from the nucleus in opposite directions (Burbidge 1967).

A property of the powerful radio sources which has only become apparent in recent years is the large amount of optical emission lying along the radio axes. This characteristic has recently been reviewed by van Breugel (1988). The extended emission is frequently line emission, the most common features being $\mathrm{H} \alpha,[0 \mathrm{III}] 5007,4959$ and Ly $\alpha$ (in objects with large enough redshifts). The sizes of the line emitting regions range from $\sim 10 \mathrm{kpc}$ up to $\sim 1 \mathrm{Mpc}$ but they are usually smaller than the radio sources. They show a variety of morphologies. Sometimes significant velocity differences of order $\leq 2000 \mathrm{~km} \mathrm{sec}^{-1}$ are seen in the gas. It appears that the extended emission requires ejection from the center, interaction with an extended gaseous medium, and an ionizing source at the center. There is also evidence in some cases for discrete optical synchrotron sources not associated with the nucleus and for young stars in some of these extended regions. Thus we have evidence that the central machine in the radio galaxy is able to eject far more than just beams of relativistic particles.

We briefly mention two examples of nearby radio galaxies which may relate through evolution to the galaxies reviewed by van Breugel.

a. NGC 5128, the radio source Centaurus A, which is only about $5 \mathrm{Mpc}$ away. It has been known for many years that while this is not a very powerful radio source $\left(\sim 10^{41}\right.$ ergsec $\left.^{-1}\right)$ it is very large, $\sim 0.75 \mathrm{Mpc}$. It has a very compact high energy jet aligned in the direction of the large radio source. Young stars are also found along the direction of the major axis (Graham and Price 1981).

Probably the most striking feature of the region around NGC 5128 is the distribution of galaxies which, as was pointed out long ago by de Vaucouleurs and by Arp (1968), also lie along the major axis determined by the inner jet and extended radio source, extending over about $2 \mathrm{Mpc}$. We believe that this general alignment is not an accident, but that it should be considered as a feature which is similar to that seen in optical emission in radio galaxies with large redshifts; i.e. what we are proposing is that what we see in powerful radio galaxies at large redshifts is the process of galaxy formation suggested by Ambartsumian, and that several billion years later such a system has evolved into the large scale structure seen around NGC 5128. Several billion years ago, 
NGC 5128 was a major source of energy, a much more powerful radio source than it is now, and it has given rise to all of the galaxies in that region.

b. M87 has an extended radio source and $\mathrm{x}$-ray source lying about it. The optical jet and counter jet and weak line emission are well known. Wade (1960) pointed out that the radio source 3C 272.1 (NGC 4374) lies some $400 \mathrm{kpc}$ from M87 exactly along the position angle of the optical jet. More recently Arp (1987) has pointed out that the discrete x-ray sources in this region and a number of galaxies also lie along the same axis. We propose that this is not an accident, but that M87 much earlier in its history was similar to the high redshift radio sources and gave rise to the other objects.

We finally mention another related phenomenon. This is the discovery of QSOs lying along the axes of powerful radio galaxies. Some examples are 3C 303 (Kronberg et al 1977), 3C 441 (McCarthy et al 1988), B2 0924+30 (Ekers et al 1975, Arp 1987), and 4C 31.32 (Bridle \& Perley 1984, Arp 1987). These also suggest that ejection involving QSOs is taking place.

\section{REFERENCES}

Ambartsumian, V.A. 1958, La Structure et l'evolution de l'univers

(Brussels, Stoops), p. 241.

Arp, H.C. 1968, Pub. A.S.P., 80,129.

Arp, H.C. 1971, Astrophys. Letters,9,1.

Arp, H.C. 1987, Quasars, Redshifts and Controversies (Interstellar Media, Berkeley, CA).

Blandford, R. and Rees, M. 1974, M.N.R.A.S.,169,395.

Bridle, A.H. and Perley, R. 1984, Ann.Rev. Astronomy \& Astrophysics, 22, 319.

Burbidge, G 1967, Nature, 216, 1287.

Burbidge, G 1979, Nature,282, 451.

Burbidge, G.R., Burbidge, E.M. and Sandage, A.R. 1963, Rev. Mod. Phys., 35, 947.

Burbidge, E.M., Burbidge, G.R., Solomon, P.M. and Strittmatter, P.A. 1971, Ap.J., 170, 233.

Ekers, R., Fanti, R., Lari, C. and Ulrich, M.-H. 1975, Nature, 258, 584.

Graham, J. and Price, R. 1981, Ap.J.,247,813.

Kippenhahn, R. and de Vries, H.L., 1974, Astrophysics \& Space Science, 26, 131.

Kronberg, P., Burbidge, E.M., Smith, H.E. and Strom, R.G. 1977, Ap.J., 218, 8.

McCarthy, P.J., Dickinson, M., Filippenko, A., Spinrad, H. and van Breugel, W. 1988, Ap.J. Lett., 328, L29.

Monk, A.S., Penston, M.V., Pettini, M. and Blades, J.C. 1986, M.N.R.A.S., 222, 787.

Monk, A.S., Penston, M.V., Pettini, M. and Blades, J.C. 1988, preprint.

Pocock, A.S., Blades, J.C., Penston, M.V. and Pettini, M. 1984, M.N.R.A.S., 210, 373.

Stocke J.T., Schneider, P., Morris, S.L., Gioia, I., Maccacaro, T. and Schild, R.E. 1987, Ap.J.,315, L11.

Sulentic, J. 1983, Ap.J. Lett., 265,L49.

van Breugel, W. 1988, preprint.

Wade, C.M. 1960, The Observatory, 80, 235. 